\title{
A novel mutation in ETFDH manifesting as severe neonatal-onset multiple acyl-CoA dehydrogenase deficiency
}

Francois $\mathrm{H}$ van der Westhuizen ${ }^{\mathrm{a} 1}$, Izelle Smuts ${ }^{\mathrm{b}}$, Engela Honey ${ }^{\mathrm{c}}$, Roan Louw ${ }^{\mathrm{a}}$, Maryke Schoonen ${ }^{\mathrm{a}}$, Lindi-Maryn Jonck ${ }^{\mathrm{a}}$, and Marli Dercksen ${ }^{\mathrm{a}}$

${ }^{\text {a}}$ Human Metabolomics, North-West University, Potchefstroom, South Africa

${ }^{b}$ Department of Paediatrics, Steve Biko Academic Hospital, University of Pretoria, South Africa

${ }^{c}$ Department of Genetics, University of Pretoria, South Africa

${ }^{1}$ Corresponding author.

E-mail: Francois.vanderWesthuizen@nwu.ac.za

Tel.: $+27(0) 182992318$

Fax: +27 (0)18 2992477

Postal Address: Human Metabolomics, Private Bag X6001, North-West University, Potchefstroom 2520, South Africa

\section{Highlights}

- A Novel homozygous mutation, c.1067G > A in ETFDH, was found in a type I MADD patient.

- Two other type III MADD patients had a compound heterozygous,

c. $1067 \mathrm{G}>\mathrm{A}+\mathrm{c} .1448 \mathrm{C}>\mathrm{T}$.

- Structural instability of ETFDH in muscle was confirmed with SDS-PAGE.

- These mutations should be screened for in South African Caucasian MADD patients.

\section{Abstract}

Neonatal-onset multiple acyl-CoA dehydrogenase deficiency (MADD type I) is an autosomal recessive disorder of the electron transfer flavoprotein function characterized by a severe clinical and biochemical phenotype, including congenital abnormalities with unresponsiveness to riboflavin treatment as distinguishing features. From a retrospective study, relying mainly on metabolic data, we have identified a novel mutation, c.1067G>A (p.Gly356Glu) in exon 8 of ETFDH, in three South African Caucasian MADD patients with this index patient presenting the hallmark features of type I MADD and two patients with compound heterozygous (c.1067G $>A+$ c.1448C $>T$ ) mutations presenting with MADD type III. SDS-PAGE western blot confirmed the significant effect of this mutation on ETFDH structural instability. The identification of this novel mutation in three families originating from the South African Afrikaner population is significant to direct screening and strategies 
for this disease, which amongst the organic acidemias routinely screened for, is relatively frequently observed in this population group.

\section{Keywords:}

Multiple acyl-CoA dehydrogenase deficiency, MADD, glutaric aciduria type II, ETFDH

\section{Introduction}

Multiple acyl-CoA dehydrogenase deficiency (MADD, OMIM: 231680), also known as glutaric aciduria type II (GAll), is an autosomal recessive metabolic disorder. Mutations in the ETFA, ETFB and ETFDH genes result in the deficient function of alpha and beta subunits of the electron transfer protein (ETF) or ETF-coenzyme $Q$ oxidoreductase, respectively. Dysfunction in either of these two flavoproteins leads to compromised fatty acid and amino acid oxidation as well as choline metabolism.

The MADD phenotype can be divided into three clinical classes: two neonatal-onset forms with (type I) or without (type II) congenital anomalies or a mild/delayed lateonset form (type III), which shows a variable degree of response to riboflavin treatment [1-3]. The neonatal-onset form has a poor prognosis, presenting within the first week(s) of life with severe hypoglycemia, mild to severe hyperammonaemia, metabolic acidosis, with or without dysmorphic features, hepatic-, cardiac- and renal involvement. The phenotypic presentation of type III is highly variable, characterized by episodic lethargy, vomiting, hypoglycemia, metabolic acidosis with or without hyperammonaemia and hepatomegaly. Painful myopathy can be progressive and abnormal lipid storage and free carnitine depletion is commonly observed $[1,3]$.

The biochemical characterization of MADD includes organic acid and acylcarnitine profiling, which indicates, increased levels of aliphatic mono- and dicarboxylic acids, acylglycine conjugates as well as an increase of C4-C18 acylcarnitines in blood [1]. Although mutations are most frequently encountered in the ETFDH gene in the lateonset form of MADD, for the most severe (type I) neonatal form of the disease mutations in ETFDH, ETFA, and ETFB are found more or less in equal frequency [2,3,5-9]

As mutation screening is not currently offered routinely in our (South African) population, and since biochemical data through routine metabolic screening suggests that MADD may be in the same order of prevalence as other organic acidemias in South African populations $[10,11]$ we've begun to retrospectively investigate the 
molecular genetics of MADD with metabolic data as the denominator. From the initial explorative investigations in three Caucasian patients, we've identified a novel pathogenic variant present in all three cases, with the homozygous form in the index case presenting as a (type I) severe neonatal onset form of the disease.

\section{Patients and methods}

Informed consent was obtained as part of current studies with ethical approval numbers 91/98 (University of Pretoria) and NW-00170-13-S1 (North-West University). Table 1 summarizes the clinical, biochemical and genetic features of the three patients.

Table 1. Summary of clinical, biochemical and genetic features of patients

\begin{tabular}{|c|c|c|c|}
\hline & Patient 1 (Index) & Patient 2 & Patient 3 \\
\hline Gender & Male & Female & Female \\
\hline Age of onset/death & Week 1/Day 9 & $\begin{array}{l}\text { Year 2/undetermined (at least } \\
\text { past } 8 \text { years) }\end{array}$ & Year 4/23 \\
\hline \multirow[t]{2}{*}{ Main clinical features } & $\begin{array}{l}\text { Intra-uterine growth retardation } \\
\text { Multiple congenital anomalies: } \\
\text { - Cardiac lesions } \\
\text { - Hydronephrosis }\end{array}$ & $\begin{array}{l}\text { Progressive muscle weakness } \\
\text { Hepatomegaly } \\
\text { Migraine } \\
\text { Exercise intolerance } \\
\text { Hypermobility }\end{array}$ & $\begin{array}{l}\text { Progressive muscle weakness } \\
\text { Hepatomegaly } \\
\text { Migraine }\end{array}$ \\
\hline & Riboflavin unresponsive & $\begin{array}{l}\text { Riboflavin responsiveness } \\
\text { undetermined }\end{array}$ & Partially responsive to riboflavin \\
\hline $\begin{array}{l}\text { Routine Biochemistry: } \\
\text { - } \quad \text { Metabolic acidosis } \\
\text { - } \quad \text { Glucose } \\
\text { - } \quad \text { Ammonia } \\
\text { - } \quad \text { Lrine ketones } \\
\text { - } \quad \text { Transataminases } \\
\text { - } \quad \text { Creatine kinase } \\
\text { - } \quad \text { CBC findings }\end{array}$ & $\begin{array}{l}\text { Present } \\
\text { Normal- } \downarrow \\
\uparrow-\uparrow \uparrow \uparrow \\
\text { Absent } \\
\text { Normal- } \uparrow \\
\uparrow \uparrow \uparrow \text { GG, AST } \\
\text { Not performed } \\
\text { Pancytopenia }\end{array}$ & $\begin{array}{l}\text { Not present } \\
\text { Normal } \\
\text { Not performed } \\
\text { Absent } \\
\uparrow \uparrow \\
\uparrow A S T, A L T \\
\text { Normal } \\
\text { Normal }\end{array}$ & $\begin{array}{l}\text { Present } \\
\text { Normal- } \downarrow \\
\uparrow-\uparrow \uparrow \uparrow \\
\text { Absent } \\
\text { Normal- } \uparrow \\
\uparrow A S T, A L T \\
\text { Normal- } \uparrow \uparrow \\
\text { Normal }\end{array}$ \\
\hline Histology & Not performed & $\begin{array}{l}\text { Muscle: cytoplasmic lipid } \\
\text { accumulation in myofibres }\end{array}$ & $\begin{array}{l}\text { Liver: macrovascular steatosis, } \\
\text { abnormal lipid storage }\end{array}$ \\
\hline \multicolumn{4}{|c|}{ Metabolic findings in urine: } \\
\hline - Organic acids & $\begin{array}{l}\uparrow \text { Ethylmalonic acid } \\
\uparrow \uparrow \uparrow \text { Dicarboxylic acids } \\
\uparrow \uparrow \uparrow \text { Glycine conjugates } \\
\uparrow \uparrow \uparrow 2-\text { Hydroxryglutaric acid } \\
\uparrow \uparrow \uparrow \text { Lactic acid } \\
\uparrow \text { Krebs cycle intermediates }\end{array}$ & $\begin{array}{l}\uparrow \text { Ethylmalonic acid } \\
\uparrow \text { Dicarboxylic acids } \\
\uparrow \uparrow \uparrow \text { Glycine conjugates } \\
\uparrow 2 \text {-Hydroxyglutaric acid }\end{array}$ & $\begin{array}{l}\uparrow \text { Ethylmalonic acid } \\
\uparrow \text { Dicarboxylic acids } \\
\uparrow \uparrow \text { Glycine conjugates } \\
\uparrow 2 \text {-Hydroxryglutaric acid } \\
\text { Normal- } \uparrow \text { Lactic acid }\end{array}$ \\
\hline - Acylcarnitines & $\begin{array}{l}\uparrow \mathrm{C} 4-, \mathrm{C} 5, \mathrm{C} 5-\mathrm{DC} \\
\text { Low free carnitine }\end{array}$ & $\begin{array}{l}\uparrow C 4-, \text { C5, C5-DC } \\
\text { Low free carnitine }\end{array}$ & $\begin{array}{l}\uparrow C 4-, \text { C5, C5-DC, C8 } \\
\text { Normal free carnitine }\end{array}$ \\
\hline - Amino acids & $\begin{array}{l}\text { General amino aciduria with } \\
\uparrow \uparrow \text { Sarcosine }\end{array}$ & $\uparrow \uparrow$ Sarcosine, $\uparrow \uparrow$ Glycine & $\uparrow$ Sarcosine, $\uparrow \uparrow$ Glycine \\
\hline $\begin{array}{l}\text { Specialized } \\
\text { investigations }\end{array}$ & None & $\begin{array}{l}\text { Muscle respiratory chain } \\
\text { enzymes deficient (CI, CIII, } \\
\text { CII+III, CIV); Muscle CoQ10 } \\
\text { levels reduced }\end{array}$ & $\begin{array}{l}\text { Oxidation of } \mathrm{C} 16 \text { and } \mathrm{C} 14 \text { fatty } \\
\text { acids reduced in fibroblasts } \\
(20 \% \text { vs. controls mean; } \\
\mathrm{C} 16: \mathrm{C} 14=0.5) \text { and } \\
\text { leukocytes }(5 \% \text { vs. controls } \\
\text { mean; } \mathrm{C} 16: \mathrm{C} 14=0.96)\end{array}$ \\
\hline $\begin{array}{l}\text { ETFDH variant/s } \\
(\mathrm{dbSNP})\end{array}$ & $\begin{array}{l}\text { c. } 1067 G>A+/+; \text { p.Gly356Glu } \\
\text { (novel) }\end{array}$ & $\begin{array}{l}\text { c. } 1067 G>A+/-; \text { p.Gly356Glu } \\
\text { c.1448C>T +/-; p.Pro483Leu } \\
\text { (377656387) }\end{array}$ & $\begin{array}{l}\text { c. } 1067 G>A+/-; \text { p.Gly356Glu } \\
\text { c. } 1448 C>T+/-; \text { p.Pro483Leu } \\
\text { (377656387) }\end{array}$ \\
\hline
\end{tabular}




\subsection{Patient 1 (index patient)}

The firstborn male child from non-consanguineous Afrikaner parents presented shortly after birth with severe metabolic decompensation unresponsive to standard metabolic intervention. He was born prematurely via cesarean section and needed admission to the intensive care immediately after delivery. A loud systolic murmur was audible and the chest $X$-ray showed a prominent right ventricle and absent pulmonary conus. The ECG displayed an extreme right QRS axis and a dysplastic pulmonary valve with a pulmonary outflow gradient measuring $57 \mathrm{mmHG}$ indicated a severe pulmonary stenosis. The foramen ovale was patent with a right to left shunt and there was a large atrial septum aneurism. The renal ultrasound showed dilated calyces in the upper lobes more severely on the left indicating hydronephrosis.

After a relative normal 24 hours complicated only with hyperlactanaemia and suspected hypothyroidism his condition rapidly deteriorated with persistent hypoglycaemia requiring high doses of intravenous glucose, metabolic acidosis and hyperammonaemia (548 $\mu \mathrm{mol} / \mathrm{L}$ ). He needed intubation and ventilation on day 6 of life but his condition deteriorated despite administration of riboflavin (200mg/kg/day) and L-carnitine $(100 \mathrm{mg} / \mathrm{kg} /$ day). He developed a bulging fontanel, sclerema neonatorum and convulsions and died on day 9 of life. GC-MS organic acid profiling performed on urine at day 3 of life showed dicarbolic aciduria with the presence of various short and medium chain acylglycine conjugates suggestive of MADD. The metabolic excretion as well as early onset was indicative of a severe neonatal phenotype with a very poor prognosis.

His family subsequently had a normal male child delivered after prenatal diagnosis performed on chorionic villi showed the fetus was heterozygous for the reported c. $1067 \mathrm{G}>\mathrm{A}$ mutation.

\section{$2.2 \quad$ Patient 2}

An eight year-old female patient presented with the complaint of clumsiness, chronic fatigue, exercise intolerance, muscle weakness and severe migrainous-like headaches. She was born at term from non-consanguineous parents and had three normal siblings. Her early gross motor development was normal and she was able to walk before the age of one year. At the age of 17 months the parents observed that she had poor head control related to muscle weakness. Since the age of three years the weakness slowly progressed. Her body mass index for her age was 13.3 and fell 
on z-score -1.8 at the age of eight years. She had a waddling gait and mild proximal muscle weakness, but she did not have a Gower sign. Polyminimyoclonus resembling spinal muscular atrophy was observed. The deep tendon reflexes were suppressed, but present. She had no other cerebellar signs. Hypermobility of all her joints was observed. Her general level of functioning was on the level of 6.5 years.

She had hepatomegaly with mildly elevated liver enzymes. Renal functions were normal, creatine kinase was normal and the lactate:pyruvate ratio was 16.3. The routine metabolic investigations showed general dicarboxylic aciduria including acylglycine and acylcarnitine conjugates. Spinal muscular atrophy was excluded: the patient did not have a homozygous deletion in the telomeric region of the survival motor neuron on exon seven. A muscle biopsy was performed for the determination of respiratory enzyme analyses, after which the patient and family was not responsive to any further follow up.

\subsection{Patient 3}

This patient was born at term from non-consanguineous parents with one healthy sibling and no previous family history of neuromuscular disease. She initially presented with hypotonia and only started to walk at the age of 20 months. The progressive myopathic phenotype of unknown cause was confirmed at the age of two year after a comprehensive clinical evaluation. Metabolic decompensation was observed at the age of three years when she was admitted with coma, severe hyperammonemia (394 $\mathrm{mol} / \mathrm{L}$ which decreased to $90 \mu \mathrm{mol} / \mathrm{L}$ after hospitalization), hepatomegaly and hypoglycemia without urinary ketones. No myoglobinuria were noted, but prerenal failure was suspected. Her liver enzymes were elevated, together with elevated creatine kinase. A liver biopsy indicated macrovascular steatosis with no necrosis. The metabolic investigations showed increased levels of urine monoand dicarboxylic acid, acylglycine as well as short and long chain acylcarnitine conjugation. The latter in combination with general clinical presentation of myopathy and liver involvement was suggestive of MADD. She received riboflavin and Lcarnitine treatment together with a low protein diet and improved partially, waking up from the coma and showing stronger motor function. Progressive deterioration of muscular function did however occur and she suffered from episodic migraines. Regular follow-up metabolic investigations were done to assess her metabolic status, which showed moderate to limited biochemical improvement after implementation of therapeutic intervention. She did not present with cognitive impairment and became 
a fully functional adult. She unfortunately died from a stroke-like episode at age 23 before the genetic diagnosis was confirmed.

\subsection{Metabolic and biochemical investigations}

Metabolic investigations were performed essentially as described before [12]. Respiratory chain enzyme activities and CoQ10 levels were analyzed in muscle homogenates (600 x g supernatants) for Patient 2 as described before [13,14] and normalized to citrate synthase (UCS). Fatty acid oxidation of $\left[9,10-{ }^{3} \mathrm{H}\right]$ palmitic $(C 16)$ and myristic (C14) acids were performed in fibroblasts and leukocytes for Patient 3 as described before [15].

\subsection{Mutation and protein analyses}

The outcomes reported here are the results from a retrospective investigation of selected cases, using next generation sequencing (NGS, Ion Torrent) of candidate genes. Primary NGS data analysis and secondary data mining were conducted using an in-house bioinformatics pipeline and web-based annotation tools such as Ensembl online VEP runner [16] and data mining tools, including GEMINI (v0.18.0) [17] OMIM, CliniVar, and available allele frequency data from the extensive Exome Aggregation Consortium (ExAC) database. The potential pathogenic variants identified and allelic distribution in all of these cases were confirmed using Sanger sequencing and, as the $\mathrm{c} .1067 \mathrm{G}>\mathrm{A}$ variant is part of a homopolymeric region, also restriction length fragment polymorphism (RFLP) analyses (Figure 2S).

SDS-PAGE/western blot analysis were used to confirm the steady state levels of muscle ETFDH and, due to the limited material available, could only be done in Patient 2. Immunodetection using the antibodies against ETFDH (Abcam, ab131376) and $\beta$-actin (Abcam ab8227) were conducted and expression quantified against total protein load using the procedures described for Bio-Rad's V3 workflow (Bio-Rad Laboratories).

\section{Results}

Table 1 summarizes the clinical, biochemical and other specialized investigations for the three cases. The following key findings for metabolic, biochemical and genetic analyses are reported. 


\subsection{Metabolic and biochemical investigations}

Routine and follow up metabolic investigations in urine throughout the history of all three cases showed some of the hallmark metabolites associated with MADD, notably dicarboxylic acids, ethylmalonic acid, glutaric acid as well as acylcarnitines(butyryl-, isovaleryl- and glutarylcarnitine) and acylglycine (hexanoyl- isobutyryl-, isovaleryl and suberylglycine) conjugates. Patient 1 had not undergone any biopsies at time of his death. Based on her clinical features, Patient 2 was early on selected to undergo a muscle biopsy for respiratory chain enzyme analyses, which revealed combined complex I, III, II+III and IV deficiencies. The follow up investigation of CoQ10 levels in $600 \times \mathrm{g}$ supernatants showed significantly reduced levels at 0.86 nmol/UCS (controls $2.680-8.470 \mathrm{nmol} / \mathrm{UCS}, \mathrm{n}=37$ ). For Patient 3 , with an early suspicion of MADD, fatty acid oxidation confirmed the functional deficiency with palmitic (C16) and myristic (C14) acid oxidations almost equally reduced in fibroblasts and leukocytes.

A

P2 C1 C2

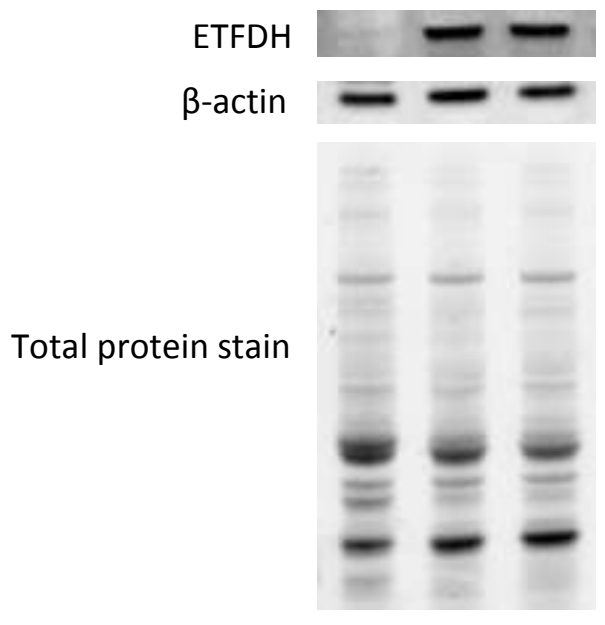

B

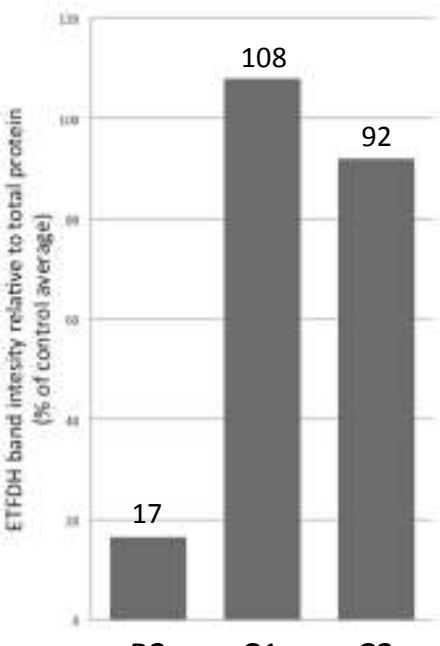

$\mathrm{P} 2$

C1

$\mathrm{C} 2$

\section{Figure 1. Structural instability of muscle ETFDH in Patient 2.}

An enriched mitochondrial preparation from muscle of Patient 2 (P2, with c.1067G>A + c. $1448 \mathrm{C}>\mathrm{T}$ variants) and two healthy controls $(\mathrm{C} 1, \mathrm{C} 2)$ were separated on SDS-PAGE and immune-stained for ETFDH and $\beta$-actin (top, Panel A). The band intensities for ETFDH were normalized to total protein content in each lane (bottom, Panel A), of which the quantified results are shown in Panel $\mathrm{B}$. 


\subsection{Mutation and protein analyses}

A c.1067G $>$ A (p.Gly356Glu) variant on the ETFDH gene was detected in homozygous form in Patient 1 (Figure 2S). This variant do not appear on any clinical or genetic databases, nor in literature consulted and was shown to be heterozygous in both parents as well as in an unaffected sibling. This novel variant is predicted to be deleterious by the SIFT prediction software and disease causing by MutationTaster prediction software. For both Patients 2 and 3 , a previously documented c.1448C>T (p.Pro483Leu) mutation and the putative deleterious c.1067G>A variant was identified in heterozygous forms. Although the patient and family of Patient 2 were lost to follow up, the carrier status for the $\mathrm{c} .1067 \mathrm{G}>\mathrm{A}$ variant was confirmed in mother and sibling for Patient 3. SDS-PAGE-western blot analysis (Figure 1) of the steady state levels for ETFDH in the only available tissue material (muscle from Patient 2) showed a significant (83\%) reduction compared to controls.

\section{Discussion and Conclusions}

Three patients presenting with clinical and metabolic features of MADD were retrospectively investigated to identify the possible genetic cause for the disease. Targeting genes possibly involved, we identified a novel pathogenic variant (c.1067G>A) in ETFDH in all three patients. This variant was homozygous in the index patient (Patient 1), who presented with a MADD type I phenotype, and compound heterozygous with the well-documented pathogenic variant $\mathrm{c} .1448 \mathrm{C}>\mathrm{T}$ in two other (Patients 2 and 3 ). Although the trajectories of clinical and further investigations were different, all three patients presented with clear clinical and metabolic features indicative of MADD type I (Patient 1), or type III (Patient 2 and 3). Patient 1, homozygous for the novel c.1067G>A variant, presented in first days of life with the most severe (type I) neonatal onset clinical and metabolic features of MADD, notably with congenital features and being unresponsive to riboflavin treatment. Patient 2, presenting with a multi-systemic neuromuscular features early in life, was initially diagnosed with a combined mitochondrial respiratory chain disorder in her ninth year. A deeper investigation into $\mathrm{Co}_{10}$ involvement and metabolomics resulted in the final MADD diagnosis and the genetic outcome presented here. Patient 3 where diagnosed with MADD early in life via metabolic and functional fatty acid oxidation tests, and were able to follow riboflavin treatment. In this case, having a variant associated with a riboflavin responsive (c.1448C $>T$ ) and unresponsive form (c.1067G>A), it is not surprising that this patient showed a partial responsiveness to riboflavin. 
A closer look at this previously undocumented c.1067G $>$ A variant, indicate a substitution of a highly conserved Glu for Gly at position 356, putatively affecting the FAD domain of ETFDH $[18,19]$. Comparing it to other type I mutations also do not highlight any evident structural similarities that can inform on any molecular basis for its pathogenicity. It is also notably the first associated with this severe phenotype identified in exon 8 of ETFDH. We had the limitation of having biological material for only one of the patients for further structural ETFDH analysis (Patient 2, with the compound heterozygous c.1067G $>A+c .1448 C>T$ variants). Considering that the homozygous c.1448C >T variant is known to result in significantly reduced ETFDH activity $( \pm 30 \%$ of WT) and steady state stability $(<10 \%$ of WT) under low riboflavin conditions [20], it can be concluded from the structural analysis (Figure 1) that the presence of the $c .1067 \mathrm{G}>\mathrm{A}$ variant on the alternative allele to the $\mathrm{c} .1448 \mathrm{C}>\mathrm{T}$ variant should have a similar impact on the instability of the native ETFDH protein. We propose that this result, in addition to the predictive parameters of the variant and the genetics in these families, support the suggestion that this novel variant can be added to the list of mutations causing a type I MADD phenotype.

Finally in conclusion, from the limited family histories and genetics that could be gathered from these three cases, no relation between these families could as yet be established. Nevertheless, we believe it is reasonable to suspect that a common founder for the $\mathrm{c} 1067 \mathrm{G}>\mathrm{A}$ mutation may have originated within this population group. The reasons for this is, firstly, that all three of these patients originate from Caucasian Afrikaner families, which is a southern African population group having descended from Europe since the $17^{\text {th }}$ century; and, secondly, that this mutation has not yet been reported in the better-screened European population groups from which the Afrikaner population originated. Considering the possibility that this mutation, in addition to the milder, riboflavin-responsive c.1448C>T mutation, may have a relatively high frequency in MADD patients of this population group, we propose that this mutation should be included in screening strategies for families with a history of MADD in this and other related population groups.

\section{Conflict of interest}

There are no conflicts of interest.

\section{Acknowledgements}

Our thanks go out to the families of patients for their assistance in this follow up study, the referring physicians, Drs M Lippert and M Engelbrecht. 


\section{Funding}

This work was in part supported by the Medical Research Council of South Africa under project title: Investigating the aetiology of South African paediatric patients diagnosed with mitochondrial disorders.

\section{References}

1. Frerman FE, Goodman SI: Defects of Electron Transfer Flavoprotein and Electron Transfer Flavoprotein-Ubiquinone Oxidoreductase: Glutaric Acidemia Type II. In The Metabolic and Molecular Bases of Inherited Disease. Edited by Scriver CR, Sly WS, Childs B, Beaudet AL, Valle D, Kinzler KW, Vogelstein B; 2001.

2. Liang WC, Ohkuma A, Hayashi YK, López LC, Hirano M, Nonaka I, Noguchi S, Chen LH, Jong YJ, Nishino I. 2009. ETFDH mutations, CoQ10 levels, and respiratory chain activities in patients with riboflavin-responsive multiple acylCoA dehydrogenase deficiency. Neuromuscul Disord 19, 212 - 216.

3. Grünert SC. 2014. Clinical and genetical heterogeneity of late-onset multiple acyl-coenzyme A dehydrogenase deficiency. Orphanet J Rare Dis 22, 117.

4. Gordon N. 2006. Glutaric aciduria types I and II. Brain \& Development 28, $136-140$.

5. Olsen RK, Olpin SE, Andresen BS, Miedzybrodzka ZH, Pourfarzam M, Merinero B, Frerman FE, Beresford MW, Dean JC, Cornelius N, Andersen O, Oldfors A, Holme E, Gregersen N, Turnbull DM, Morris AA. 2007. ETFDH mutations as a major cause of riboflavin-responsive multiple acyl-CoA dehydrogenation deficiency. Brain 130, 2045 - 2054.

6. Yotsumoto Y, Hasegawa Y, Fukuda S, Kobayashi H, Endo M, Fukao T, Yamaguchi S. 2011. Clinical and molecular investigations of Japanese cases of glutaric acidemia type 2. Mol Genet Metab 94, $61-67$.

7. Xi J, Wen B, Lin J, Zhu W, Luo S, Zhao C, Li D, Lin P, Lu J, Yan C. 2014. Clinical features and ETFDH mutation spectrum in a cohort of 90 Chinese patients with late-onset multiple acyl-CoA dehydrogenase deficiency. $J$ Inherit Metab Dis 37, 399 - 404.

8. Yamada K, Kobayashi H, Bo R, Takahashi T, Purevsuren J, Hasegawa Y, Taketani T, Fukuda S, Ohkubo T, Yokota T, Watanabe M, Tsunemi T, Mizusawa $H$, Takuma $H$, Shioya A, Ishii A, Tamaoka A, Shigematsu $Y$, Sugie $\mathrm{H}$, Yamaguchi S. 2015. Clinical, biochemical and molecular investigation of adult-onset glutaric acidemia type II: Characteristics in comparison with pediatric cases. Brain Dev 38, 293 - 301. 
9. Fu HX, Liu XY, Wang ZQ, Jin M, Wang DN, He JJ, Lin MT, Wang N. 2016. Significant clinical heterogeneity with similar ETFDH genotype in three Chinese patients with late-onset multiple acyl-CoA dehydrogenase deficiency. Neurol Sci 37, 1099 - 1105.

10. Dercksen M, Duran M, ljlst L, Mienie LJ, Reinecke CJ, Ruiter JP, Waterham HR, Wanders RJ. 2012. Clinical variability of isovaleric acidemia in a genetically homogeneous population. J Inherit Metab Dis 35, 1021 - 1029.

11. van der Watt G, Owen EP, Berman P, Meldau S, Watermeyer N, Olpin SE, Manning NJ, Baumgarten I, Leisegang F, Henderson H. 2010. Glutaric aciduria type 1 in South Africa-high incidence of glutaryl-CoA dehydrogenase deficiency in black South Africans. Mol Genet Metab 101, 178 - 182.

12. Reinecke CJ, Koekemoer G, van der Westhuizen FH, Louw R, Lindeque JZ, Mienie LJ, Smuts I. 2012. Metabolomics of urinary organic acids in respiratory chain deficiencies. Metabolomics 8, $264-283$.

13. Smuts I, Louw R, du Toit H, Klopper B, Mienie LJ, van der Westhuizen FH. 2010. An overview of a South African cohort of patients with mitochondrial disorders. J Inherit Metab Dis 33 Suppl 3: S95 -104.

14. Itkonen O, Suomalainen A, Turpeinen U. 2013. Mitochondrial coenzyme Q10 determination by isotope-dilution liquid chromatography-tandem mass spectrometry. Clin Chem 59, 1260 - 1267.

15. Brivet M, Slama A, Saudubray JM, Legrand A, Lemonnier A. 1995. Rapid diagnosis of long chain and medium chain fatty acid oxidation disorders using lymphocytes. Ann Clin Biochem 32, 154 - 159.

16. McLaren W, Gil L, Hunt SE, Riat HS, Ritchie GR, Thormann A, Flicek P, Cunningham F. 2016. The Ensembl Variant Effect Predictor. Genome Biol 17, 122.

17. Paila U, Chapman, BA, Kirchner R, Quinlan AR. 2013. GEMINI: integrative exploration of genetic variation and genome annotations. PLoS Comput Biol 9, e1003153.

18. Zhang J, Frerman FE, Kim JJ. 2006. Structure of electron transfer flavoprotein-ubiquinone oxidoreductase and electron transfer to the mitochondrial ubiquinone pool. Proc Natl Acad Sci USA.103, 16212 - 16217.

19. Watmough NJ, Frerman FE. 2010. The electron transfer flavoprotein: ubiquinone oxidoreductases. Biochim Biophys Acta 1797, 1910 - 1916.

20. Cornelius N, Frerman FE, Corydon TJ, Palmfeldt J, Bross P, Gregersen N, Olsen RK. 2012. Molecular mechanisms of riboflavin responsiveness in 
patients with ETF-QO variations and multiple acyl-CoA dehydrogenation deficiency. Hum Mol Genet 21, 3435 - 3448.

21. Kjaergaard S, Graem N, Larsen T, Skovby F. 1998. Recurrent fetal polycystic kidneys associated with glutaric aciduria type II. APMIS 106, 1188 - 1193.

22. Böhm N, Uy J, Kiessling M, Lehnert W. 1982. Multiple Acyl-CoA dehydrogenation deficiency (glutaric aciduria type II), congenital polycystic kidneys, and symmetric warty dysplasia of the cerebral cortex in two newborn brothers. II. Morphology and pathogenesis. Eur J Pediatr 139: 60 - 65.

23. Lehnert W, Wendel U, Lindenmaier S, Böhm N. 1982. Multiple Acyl-CoA dehydrogenation deficiency (glutaric aciduria type II), congenital polycystic kidneys, and symmetric warty dysplasia of the cerebral cortex in two newborn brothers. I. Clinical, metabolical, and biochemical findings. Eur J Pediatr 139: $56-59$.

24. Sudo Y, Sasaki A, Wakabayashi T, Numakura C, Hayasaka K. 2015. A novel ETFB mutation in a patient with glutaric aciduria type II. Hum Genome Var 1, 15016. 


\section{Supplementary material}

A

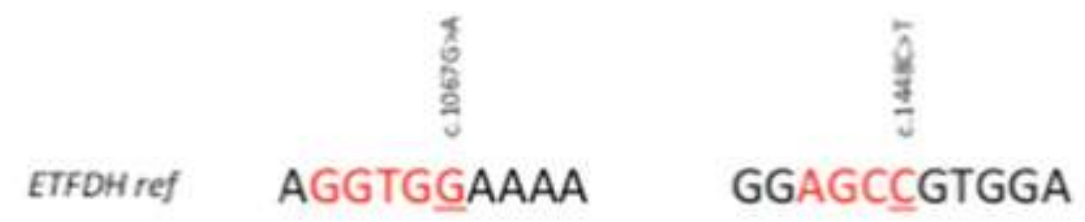

Patient 1

c.10676>A-/e

AGGTGAAAAA

Patient 2

AGGTGRAAAA

GGAGCYGTGGA

c. $1067634 \%$
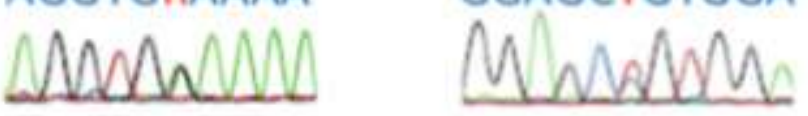

Patient 3

AGGTGRAAAA

GGAGCYGTGGA

c10670xAt/

c. $14480 \mathrm{~T}+\%$
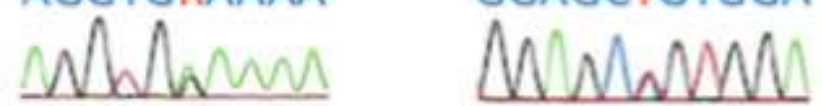

B

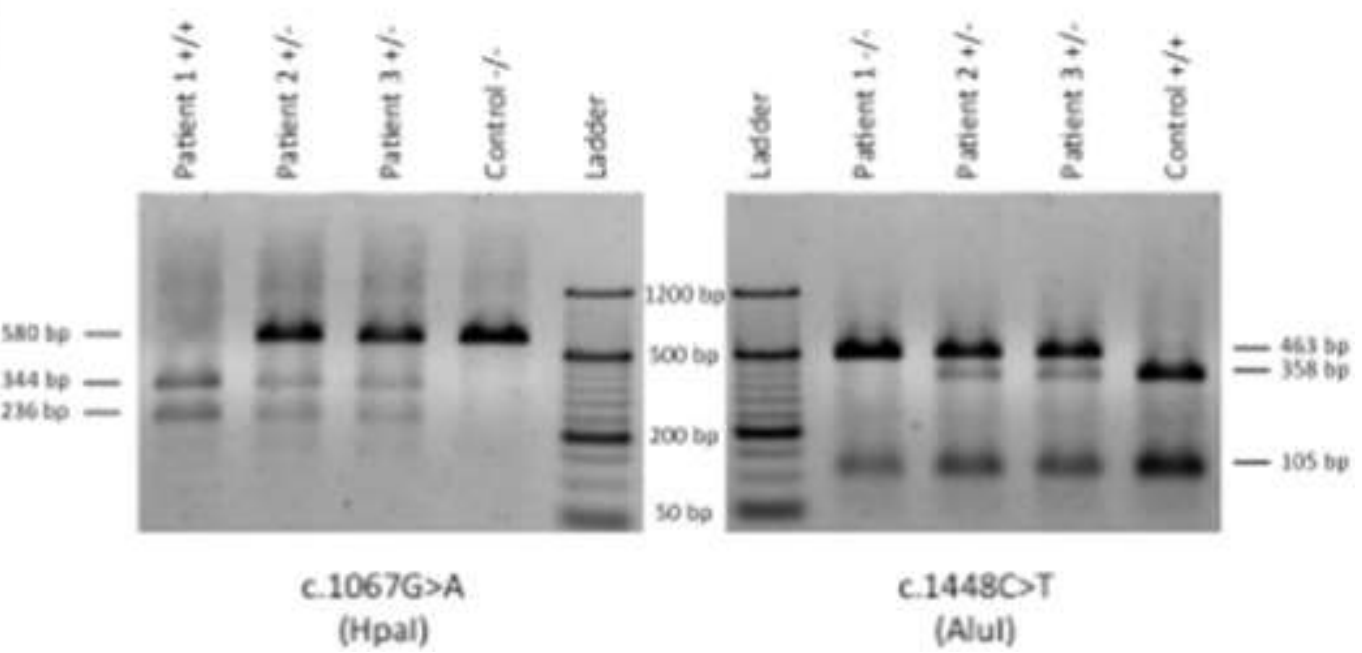

Fig. 2S. Partial sequence alignment of sequence data (A) and RFLP analysis (B) of patients.

In Panel A the sequence validation data for the three patients is shown in alignment with the reference sequence for the ETFDH gene (ENS00000171503) at the positions for the c.1067G > A and C.1448C > T mutations. $R$ indicates a heterozygous $G$ to $A$ nucleotide base change and $\mathrm{Y}$ a heterozygous $\mathrm{C}$ to $\mathrm{T}$ nucleotide base change. In Panel $\mathrm{B}$ the results from restriction fragment length polymorphism (RFLP) analyses for the patients and appropriate controls are shown. For this, DNA was first amplified using PCR and selected primers for a 580 bp region covering the c.1067G > A mutation (fwd: GCACATAGTGCTCCAAATAC; rev: CATGCCTGGCTAATCTTTCC) and a 568 bp region covering the c.1448C > T mutation (fwd: CACACATTTGGGCAGTTTCG; rev: AAACTGATCTGTCCATCGGG), respectively. The resulting fragments were digested with Hpal (GGTGA(N8)) and Alul (AGCT), as indicated in red for the two mutations in Panel A. In both cases the enzymes only cut at the positions when the mutations are present. (For interpretation of the references to colour in this figure legend, the reader is referred to the web version of this article.) 